\title{
Exclusions, Explanations, and Exceptions: On the Causal and Lawlike Status of the Competitive Exclusion Principle
}

\author{
Jani Raerinne $e^{\S}$ and Jan Baedke ${ }^{\ddagger}$
}

\begin{abstract}
The lawlike and explanatory status of ecologists' Competitive Exclusion Principle (CEP) is a debated topic. It has been argued that the CEP is a ceteris paribus law, a non-lawlike regularity riddled with exceptions, a tautology, a causal regularity, and so on. We argue that the CEP is an empirically respectful and testable strict law that is not riddled with genuine exceptions. Moreover, we argue that the CEP is not a causal explanans in explanations, because it is a coexistence law, not a causal law. Rather than being an explanans, the CEP acts as a contrastive principle sharpening causal explanations. These results contrast with previous analyses of the CEP by Eliot (2011) and Weber (1999), which are also discussed. As a more general conclusion, we suggest that accounts of causal explanation in biology have neglected some of the roles that non-causal laws play in restricting, sharpening, and facilitating causal explanations.
\end{abstract}

\section{KEYWORDS}

causality $\bullet$ ceteris paribus $\bullet$ competition $\bullet$ ecology $\bullet$ exceptions $\bullet$ laws

\section{Introduction}

The basic idea behind the Competitive Exclusion Principle (CEP) is that species that have similar or identical niches cannot stably coexist in the same place for long periods of time when their common resources are limiting. A more exact definition of the CEP states that, in equilibrium, $n$ number of sympatric species competing for a common set of limiting resources cannot stably coexist indefinitely on fewer than $n$ number of resources. The magnitude or intensity of competition between species is proportional to the degree of overlap in their niches or use of resources. Species that stably coexist do so because there are "important differences" in their niches or use of common limiting resources.

According to the modern concept of niche, the resource use of a species defines its niche. Resources can be defined as depletable or potentially limiting abiotic and biotic factors, such as mating territory, living space, or nutrients that are consumed by a species and are essential to its development, survival, or reproduction. In contrast to the large number of potential factors consistent with this definition, competition theory and the CEP are concerned only with a subset of these factors, i.e., those which are both common and limiting to competing species. There are superabundant resources, such as the amount of oxygen in the air

\footnotetext{
$\S$ Department of Philosophy, History, Culture and Art Studies, University of Helsinki P.O. Box 59, Unioninkatu 38, FI-00014 Helsinki, Finland

E-mail: jani.raerinne@helsinki.fi

‡ Department of Philosophy I, Ruhr University Bochum, Universitätsstr. 150, 44801 Bochum, Germany E-mail: jan.baedke@rub.de
} 
for terrestrial organisms, which are typically not limiting as resources and for which there is no competition within or between species. The ecology of species and the nature and renewability of resources determine, on a case-by-case basis, which of the resources are common and limiting to a species and, thus, of interest to the CEP.

An understanding of competition theory is central to clarifying how ecology as a science is organized, how it has advanced, and how explanations in this discipline proceed. In the last century, population and community ecology were dominated by the idea that competition was the explanatory force behind population and species distribution patterns, community structure, and so on. In this context, an understanding of the CEP's lawlike and explanatory nature is important, because the CEP is the central organizing principle in competition theory. From a historical perspective, it played a crucial dual role in model building; competition models, at least initially, were built so that they were consistent with the CEP (e.g., classical Lotka-Volterra competition models). The CEP thus confined model building by putting restrictions on the assumptions of models, such as the environment is not spatially or temporally heterogeneous or there is no migration or predation.

Although the CEP sometimes predicts the opposite of what is observed in nature, for instance, on the diversity of communities and life in general (Hutchinson 1959), the principle is pivotal to environmental issues and conservation biology as well. If the CEP held universally, then the diversity of life would be much less than it is, because superior competitors would always drive inferior competitors to local extinctions. Thus, it has become important to investigate theoretically (and empirically) the conditions under which the CEP does not hold, i.e., under which it has exceptions. Here, the CEP acted heuristically, suggesting which assumptions of competition models should be relaxed when applied to conditions under which the CEP does not hold: models of heterogeneous environments were suggested, higher-level interactions between species were investigated in models, and so on. It might be suggested that the CEP is a priori true in the models satisfying its basic assumptions. We provide an alternative reason why the CEP acts as a restrictive and heuristic principle in model building: the CEP is an empirically respectful and testable nomic principle.

The lawlike and explanatory status of the CEP is a debated topic. The CEP has been argued to be a tautology, a ceteris paribus law, a causal regularity, an analytical statement, and so on. Recently, Eliot (2011) suggested that the CEP should not be interpreted as a lawlike regularity nor as a causal regularity, in contrast to Weber (1999), who suggested that the CEP be considered a causal ceteris paribus law, the reason being that, according to Eliot, the principle is riddled with exceptions which are unexplainable. We review the positions taken by Eliot and Weber by arguing that the CEP can be interpreted as a strict coexistence law, one not riddled with exceptions, contrary to the opinions of both Weber and Eliot.

We proceed as follows. In sections 2 and 3, we distinguish between genuine and apparent exceptions to a regularity to show that Eliot mistakes the CEP as being riddled with genuine exceptions, whereas it is riddled with apparent exceptions. Apparent exceptions are instances to which the law or regularity does not apply. Thus, the exceptions do not represent falsifying or disconfirming instances of the law. We also argue that the CEP is a strict law rather than a ceteris paribus law. In section 4, we argue that causal regularities should remain invariant under interventions. In section 5, we discuss the causal explanatory status of the CEP and competition theory, arguing that the CEP is a coexistence law, not an invariant causal regularity. In addition, issues concerning the explanatory power of lower- and higher-level explanantia of exceptions to the CEP are discussed.

Eliot (2011) suggested that there is a novel non-nomothetic mode of explanation, called channeling explanation, that functions in competition theory. In section 6, we offer a reconstruction of Eliot's channeling explanation by showing how it is compatible with an account of causal explanation, in which both explanandum and explanans are contrastive. Moreover, we present a positive explanatory role for the CEP, which is not given by Eliot: rather than being an explanans in a causal explanation, the CEP works as a nomic principle in causal explanations, which sharpens these explanations. Finally, this suggests more generally that accounts of causal explanation in biology have neglected some of the roles that non-causal laws play in causal explanations by facilitating, sharpening, or constraining these explanations. 


\section{Genuine and Apparent Exceptions}

The general opinion is that there are many exceptions to the CEP. For instance, in a classical article "The Paradox of the Plankton," Hutchinson (1961) reported that there are many phytoplankton species living in bodies of water, despite the fact that the common resources of these species are few and very scarce, and thus limiting. Given this, one would expect that the competitive dominant species would drive other species into local extinctions. However, this was not the case, because phytoplankton species coexisted for long periods of time without local extinctions.

In contrast to ecologists' steadily increasing interest in exceptions to the CEP, the philosophical analysis of these exceptions has suffered from vague terminology. We claim that not all negative instances of a regularity are equally damaging to its lawlike status and/or truth. Therefore, let us distinguish between apparent and genuine exceptions. An apparent exception is a prima facie exception to a regularity, which is an instance in which the regularity does not apply. The ceteris paribus law literature provides a formal way to express the idea of apparent exceptions.

Let us suppose we have a generalization, such as "All F's are G's," which is qualified by a ceteris paribus (ср) clause. A cp clause is a protective proviso, such that the law holds "when some other unknown conditions remain absent and/or the same" and that prescribes the domain of a law outside of which the law does not necessarily hold. When the cp clause of a cp law is satisfied, then the law applies to a given situation, but when it is not satisfied, then the law does not necessarily apply to that situation. Let us suppose a cp clause is attached to the antecedent of the lawlike statement, $\forall x(($ Fx\&cp $) \rightarrow G x) .{ }^{1}$ An apparent exception is an instance in which the antecedent of the statement does not hold, because the cp clause does not hold. The law does not apply because the other conditions were not equal, did not remain unchanged, etc. In other words, an apparent exception is an instance of (Fa\& $\neg \mathrm{cp}$ ), which shows that the antecedent of $\forall x((F x \& c p) \rightarrow G x)$ does not hold. Consequently, the fact that there is an instance ( $\neg \mathrm{Ga})$ does not matter, because the law applies only apparently to the situation. An apparent exception is neither an exception to nor an instance of the statement, because the statement does not apply to cases in which the cp condition, or the antecedent in general, does not hold. Trivially, a case in which a law does not hold is not a case which could show that the law is false.

Genuine exceptions are instances of the form ((Fa\&cp) \& ( $\neg \mathrm{Ga})$ ) of the former statement in which the law applies, because the antecedent is true. But since the consequent is false, the entire law statement is false, and consequently, the above instance is a real exception to and a falsifying or disconfirming instance of the statement $\forall x(($ Fx\&cp $) \rightarrow G x)$.

For example, linkage provides an apparent exception to Mendel's rule of independent assortment. In linkage, genes that do not assort independently of one another during the formation of gametes are located on the same chromosome. However, the rule of independent assortment applies only to those genes located on different homologous chromosomes. The Titus-Bode law, which was formulated in the 18th century, is a generalization concerning the regular spacing of the orbits of planets. The orbit of Neptune provides a genuine exception to the law, showing that the law is false.

Ceteris paribus clauses cover the absence or lack of change in the interfering conditions that are responsible for the law not applying under some conditions. The cp clause of a cp law should not be eliminable by a complete and finite list of known conditions $\left(\mathrm{Ix}_{\mathrm{i}}\right)$ that could interfere with the law. In most cases, such a list cannot be made, either because we do not know all the interfering conditions or because their number is so high or heterogeneous in nature that the elimination of cp clauses becomes impossible. Yet if such an elimination or listing of conditions can be made and is carried out, then this amounts to a "lazy meaning" of cp laws. In this case, we have a law that is strictly complemented and all of its interfering conditions $\left(\mathrm{Ix}_{\mathrm{i}}\right)$ are mentioned, for instance, in a conjunct of their negations in the antecedent of the law statement, $\forall \mathrm{x}\left(\left(\mathrm{Fx} \&\left(\neg \mathrm{Ix}_{1} \& \neg \mathrm{Ix}_{2} \& \neg \mathrm{Ix}_{3} \& \ldots \& \neg \neg \mathrm{Ix}_{\mathrm{n}}\right)\right) \rightarrow \mathrm{Gx}\right)$. This is a law in which conditions of applicability are fully specified, whereby the law applies to cases in which the listed known conditions ( $\left.\mathrm{Ix}_{\mathrm{i}}\right)$ are absent. Strictly complemented laws are strict laws, and their problems are not those of cp laws.

The central problem in accounts of cp laws is to provide cp clauses with semantics. The problem involves not only providing formal truth conditions for laws, but also providing cp clauses with definite content and meaning that distinguish the legitimate uses of cp clauses from illegitimate and ad hoc uses. 
Without such semantics, we would not be able to tell whether a law applies to a given situation. In other words, we need an idea of how apparent exceptions can be distinguished from genuine exceptions. A related concern is that cp clauses make generalizations empirically vacuous. Empirically vacuous generalizations make trivially true or ad hoc claims, such as "All F's are G's, except when they are not," which are immune to empirical testing and disconfirmation. Empirically vacuous generalizations also lack explanatory and predictive power.

Many philosophers and scientists maintain that, if an exception to a regularity can be explained independently and in a non-ad-hoc way, then the exception is apparent, not genuine (Pietroski \& Rey 1995). In other words, apparent exceptions are explainable, whereas genuine exceptions are not. A related idea is that explanations of apparent exceptions to cp laws in the special sciences come from lower levels of mechanisms and/or are stated in the vocabulary of lower-level sciences (Davidson 1970; Fodor 1991; Carrier 1998).

\section{The CEP as a Strict Law}

When faced with a situation that seems to be an exception to the CEP, as happens with a guild of birds of prey that hunt the same food, such as voles of the genus Microtus, an ecologist undertakes a closer investigation of the situation and the ecology of the species. It is not uncommon that species are found to be less similar with respect to their niches than they initially seemed (Lack 1945, 1946; Heatwole \& Davis 1965). Thus, what appeared to be a genuine exception to the CEP is found to be an apparent exception, since the principle does not apply to cases in which there are important differences between the niches of competing species.

Similarly, environmental heterogeneity can alter the competitive rankings between species, so that the species do not reach their competitive equilibrium in which dominant species drive other species into local extinctions. This was the explanation that Hutchinson (1961) gave for his paradox of the plankton discussed above. In high latitudes, there are temporal or seasonal variations of limiting resources of many species and guilds: the amount of sunlight, minerals, nutrients, etc. If the temporal variation of resources is faster - yet not too fast - than the time needed for competitive systems to reach their equilibria, then the temporal variation of resources can prevent competitive systems from reaching their equilibria. Thus, one (or more) species is locally not outcompeted and displaced by (an)other ecologically more efficient species. Spatial environmental heterogeneity works differently. There may be poor-quality habitat patches that provide refugee camps for an inferior competitor species and from which they recolonize the high-quality patches where they have been displaced by a better competitor. If vagility or the intrinsic growth rate of the former species is sufficiently high and/or it has a broader environmental tolerance, this can lead to the coexistence of competitor species, despite the fact that the other species is superior with regard to competition and use of resources. Therefore, the various instances of environmental heterogeneity have a robust result; they lead to apparent exceptions to the CEP and thus make coexistence possible. The CEP does not hold in cases such as these, because the coexistence of competitors is not stable.

Many other empirical and theoretical studies of various so-called coexistence mechanisms understand exceptions to the CEP as apparent, not genuine (Gilbert et al. 1952; Utida 1953; Slobodkin 1964; Amarasekare 2007; Tang \& Zhou 2011). We suggest that these coexistence mechanisms are of two kinds: intervening and constitutive.

By a constitutive coexistence mechanism, we mean explanations of exceptions to the CEP that show that a certain system does not constitute or realize a competitive system, despite initial appearances. Coexistence of competitors is possible and explained, because the competitive system is only an apparent, not a genuine, system, as there are important ecological differences between the species that allow coexistence. An explanation showing that the niches of competing species are less similar than they appear is an instance of a constitutive coexistence mechanism, because the ecological differences between species promote the coexistence of apparent competitors (Lack 1945, 1946; Heatwole \& Davis 1965). For $n$ number of species competing for less than $n$ number of common and limiting resources, variance at resource levels, such as an unpredictable amount of rainfall in arid environments, can itself work as a new resource (Tilman 1986). In this case, we are again dealing with an apparent competitive system, because the number of species is not less than their common limiting resources. Alternatively, the situation can be described, as one in which 
there are important ecological differences between species' niches, with the effect that some species are more capable than others of utilizing variance at resource levels.

In contrast, an intervening coexistence mechanism interferes with a system that constitutes a real competitive system, but prevents competitive exclusion from occurring. This type of interference affects the system in such a way that its capacity for competitive exclusion is not manifested because it affects, for instance, the triggering conditions of the system's capacity or prevents the system from reaching equilibrium. A common predator or parasite of competitor species can rarify the population densities of competing species, so that the densities remain beneath their environment's carrying capacity (Utida 1953; Slobodkin 1964; Paine 1966). In such a situation, there is a real competitive system with a capacity for competitive exclusion. But this capacity does not manifest itself, because the common resources are not limiting, owing to interference from outside the competitive system that rarifies the populations of competing species below their environment's carrying capacity, at which point resources begin to be in short supply. Environmental heterogeneity and intermediate disturbance are other examples of intervening coexistence mechanisms accounting for apparent exceptions to the CEP.

The Intermediate Disturbance rule states that intermediate levels of disturbances, such as keystone predators or pathogens, are capable of mediating the coexistence of competitor species, thus maintaining the local diversity of a community at a high level (Paine 1966; Durán \& Castilla 1989). In other words, local species diversity often peaks at the intermediate levels of habitat disturbances. Intermediate disturbances have the same effect, for instance, through reduction of population densities of competitor species, which counters the strong competitive effects between species and provides apparent exceptions to the CEP. Too small or infrequent disturbances lead to local extinctions of competitively inferior species by competitively dominant species (i.e., the CEP holds), whereas too intense or frequent disturbances allow the few species that are the most stress-tolerant to exclude other species from a habitat (i.e., there is another apparent exception to the CEP).

Weber (1999) and Eliot (2011) did not explicitly distinguish between apparent and genuine exceptions, but spoke vaguely of the CEP as being riddled with exceptions. Weber's idea seemed to be that the exceptions were apparent ones, whereas Eliot seemed to think that the CEP was riddled with unsystematic and unexplainable exceptions, i.e., genuine exceptions. One of our arguments is that the distinction should be made explicit, because it matters: the CEP is riddled only with apparent exceptions, systematically explainable by constitutive and intervening coexistence mechanisms, which are thus cases to which the law does not apply. However, our position does not coincide with Weber's either.

As far as we know, there is only one putative genuine exception to the CEP, whereas the other exceptions seem to be explainable as apparent by means of the two coexistence mechanisms discussed. However, even this particular case is likely to be an apparent exception to the CEP. We refer to the famous competition laboratory experiments with two phylogenetically and ecologically similar flour beetles of the genus Tribolium by Park and his colleagues (Leslie et al. 1968).

Park's $(1948,1968)$ previous laboratory experiments showed that when populations of two flour beetles ( $T$. confusum and $T$. castaneum) compete for a common limiting resource and when the initial and boundary conditions of the experimental competitive systems, such as the initial population number of the species, the amount of their common resource, and the level of infection by a common parasite (Adelina tribolii) were varied, it is not always possible to predict in advance which of the species will outcompete the other in a specific experimental setup. Moreover, the time required for the system to reach its competitive equilibrium, i.e., the extinction of one or another of the species, varied by almost an order of magnitude and often covaried with the variance of the initial and boundary conditions. Coexistence did not occur in any of Park's previous experiments, but one species invariably displaced another in competition, as required by the CEP. Park's later studies were designed so that he tried to control for and vary different kinds of initial and boundary abiotic and biotic conditions to determine how they affected the fate of the competitive systems.

The study by Leslie et al. (1968) investigated the effect of varying the initial number of Tribolium species populations on the outcome of competition. This was deemed important, since previous studies suggested that $T$. castaneum would outcompete $T$. confusum in all of the competitive systems and under all initial and boundary conditions, owing to its ecological advantages over $T$. confusum. For instance, laboratory strains of $T$. castaneum were found to have (evolved) higher intrinsic growth rates and were more voracious predators than $T$. confusum, which led to $T$. castaneum being considered a superior competitor. 
The exceptional competitive system, as an experiment, began with a numerical advantage to the less superior competitor: 32 individuals of $T$. confusum vs. four individuals of $T$. castaneum. The exceptional competitive system initially proceeded as expected: $T$. castaneum appeared to outcompete and displace $T$. confusum from the system, since its population numbers began to rise, whereas those of $T$. confusum started to decline, but then $T$. confusum managed to recover its population numbers, so that the two species coexisted. The coexistence lasted for a long time, given that the generation time of these organisms is a few weeks, and the system was terminated by the researchers when it had run for 960 days. Later, the researchers initiated new experiments with the populations of the exceptional competitive system. The design of these series B experiments was identical to that of the original experiments. Nothing in the series B experiments was exceptional, however; they ran as expected. Moreover, the usual winner of the competition was $T$. castaneum.

There were valid reasons for Leslie et al. (1968) to doubt that the exceptional competitive system represented a genuine exception to the CEP. First, the series B experiments provided no replication of the previous result, but rather verified the CEP, which casts doubt on the idea that the exceptional system was a genuine exception to the CEP. Instead, the series B results suggest that the exceptional competitive system was either an artifact of the experimental settings or explainable by means of coexistence mechanisms. Second, the authors developed a discrete-time stochastic model to simulate the population dynamics of the Tribolium competitive system. The results of the model fit well with the experimental results and data. More important, the model suggested that the time required for the superior competitor to outcompete the other in the system could be as long as 1,120 days, which is longer than the time the exceptional system was investigated. Thus, both the experimental and the theoretical data suggested that the case was an apparent exception to the CEP, one of prolonged competitive exclusion, rather than a genuine exception.

We do not argue that the CEP lacks any genuine exceptions, because the issue is at least partly empirical rather than philosophical. However, we do have an argument that shows that the principle is not riddled with genuine and unsystematic exceptions, but rather that it has systematic apparent exceptions, which are explainable using proprietary and distinctively ecological terminology. This at least is evidence for the claim that the CEP may be a universally true strict law rather than a ceteris paribus law (contra Weber 1999) and that ecologists have systematic theoretical resources to explain the exceptions to the CEP as apparent exceptions (contra Eliot 2011: 6-7).

There are many natural interfering conditions, such as earthquakes, fires, and storms, which are capable of producing exceptions to the CEP. Let us mark with an "I" conditions interfering with the CEP that are physical, chemical, geological, and so on in nature, i.e., conditions that come from the lower levels. In fact, it does not matter whether the I's come from levels higher than biology, such as human interventions. The point is that the I's are interfering conditions that are outside the domain of biology, and moreover, there are numerous I's capable of producing exceptions to the CEP, which threaten its validity and/or suggest that it may be a ceteris paribus law. ${ }^{2}$

The I's could variously affect competitive systems and their components. However, they do not produce unsystematic genuine exceptions to the CEP, but can be systematically explained as apparent exceptions by coexistence mechanisms. We can provide the explanations in terms of proprietary ecological explanantia rather than in terms of lower-level theory. Below, we use italics to highlight the explanations of exceptions to the CEP given in ecological explanantia.

The I's may affect the limiting resources of species and result in the coexistence of competitors that would not otherwise have coexisted. The I's may make certain limiting resources superabundant (e.g., human interventions/geological conditions that result in the eutrophication of water). This can be explained, however, as a case in which there is luxury or hoarding competition, i.e., the resources were no longer limiting. The exception to the CEP by the I's is thus an apparent exception. The I's may negatively affect the population densities of competing species (earthquakes, etc.) and result in coexistence that would not have occurred without the I's. This could be explained as an apparent exception to the principle: the species are not at their environment's carrying capacity and/or the resources are not limiting. The I's could eliminate some of the species from the system (earthquakes, storms, etc.) and a coexistence of competitors may follow. If, as a consequence, the number of species in the competitive system is fewer in number than the common limiting resources of the species, then the exception would be explainable: the competitive system is only an apparent competitive system. 
The I's could affect the variance in resource levels (climatic and weather conditions, among other things) and result in competitive coexistence that would not have occurred without the I's. We have already given reasons for how this can be explained as an apparent exception to the principle: the coexistence occurred because the niches of the species are different and/or the variance in resource levels counts as a new resource itself. The I's could affect the environment such that it becomes spatially or temporally heterogeneous (earthquakes, storms, fires, climatic and weather conditions, etc.), resulting in coexistence that would not have occurred without the I's. Again, this can be explained by claiming that the competitive rankings between species change so that a competitive equilibrium is not reached and the coexistence of competitors is not stable, in the sense of Hutchinson (1961) discussed above. Thus, there seem to be good reasons to believe that all sorts of I's - lower- or higher-level ones along with those within the domain of ecology and those that are not - can be explained as producing systematic apparent exceptions to the CEP, using proprietary and distinctive ecological terminology.

Proponents of ceteris paribus laws typically hold that the explanations for exceptions to regularities in the special sciences and the specification of their application domains come from lower levels of mechanisms and/or are stated in the vocabulary of the lower-level sciences (Davidson 1970; Fodor 1991; Carrier 1998). However, in the case of the CEP, ecologists are capable of explaining exceptions using distinctive and proprietary ecological concepts, such as common predators, differences in niches or resource use, and non-limiting resources, which are apparent exceptions to the principle. There is no need to refer to the lower-level sciences when ecologists employ intervening and constitutive coexistence mechanisms as explanantia for the exceptions to the CEP and specify under what conditions the principle should hold. Thus, according to some prominent accounts of cp laws, the CEP does not count as a cp law.

The problem of ceteris paribus laws is providing truth conditions to the proviso clause "whenever some other unknown background conditions remain absent and/or the same," which prescribe the domain of a law outside of which the law does not necessarily hold. If the interfering background conditions were known in advance, then there would be no need for ceteris paribus clauses nor any problem of semantics for ceteris paribus laws. In other words, there would be only an epistemic problem of determining what known background conditions could interfere with a law, and with regard to the CEP, there is an epistemic, not a semantic, problem because we are familiar with the various mechanisms producing apparent exceptions to the principle, namely, intervening and constitutive coexistence mechanisms, as discussed above.

In other words, we argue that the CEP has only the appearance of a cp law, while in fact, it is a strict law, because it can be strictly complemented (contra Weber 1999). Pietroski and Rey (1995: 82) characterized the central problem of cp laws - vacuity - and their solution as follows: "[Ceteris paribus] clauses are 'cheques' written on the banks of independent theories ... which determine whether the cheque can be cashed" (82). "These cheques represent a 'promise' to the effect that all ... [negative] instances of the putative law in question can be explained by citing factors that are ... independent of that law" (89).

In the CEP, however, we do not have to write checks, because we already have cash in our pockets: the CEP holds except when intervening and constitutive coexistence mechanisms are in place. There is no empirical vacuity, semantic inaccuracy, ad hocness, or open-endedness in the proviso clause of the CEP, because we already know two mechanisms capable of producing apparent exceptions to the CEP. There is only the epistemic problem of determining which of the different instances of known exception-making mechanisms is responsible for exceptional instances in the CEP, not a semantic problem of spelling out in detail the proviso clause of the CEP in terms of cp clauses. The proviso clause of the CEP can be eliminated by a known and finite list of factors or by conditions capable of producing apparent exceptions to the CEP. Thus, the CEP can be strictly complemented. It is a strict law in disguise, which is not riddled with genuine exceptions, but same-level explainable apparent exceptions, suggesting that the principle may even be universally true.

Eliot (2011) criticized Weber (1999) as being too optimistic in thinking that ecologists have theoretical resources to explain away the exceptions to the CEP: "But ... that we anticipate in advance some exceptions or domain restrictions is beside the point; if we are interested in lawfulness ..., we want to know whether all the limitations on the CEP can be specified in advance. Otherwise it is a classically problematic ceteris paribus generalization" (Eliot 2011: 6; original emphasis). While it is not exactly clear to us what Eliot's demand amounts to, we take it that the above can be understood in two ways.

\section{昂}


The first way is to demand that there is an (a priori or philosophical) argument showing that the CEP lacks any genuine exceptions in every place and at every time. This is not what Eliot seems to be requiring, however. He believes - and we share his view - that the CEP is an empirical regularity and consequently, it should remain an open empirical and scientific question of whether a genuine exception to the principle can be found. It would be a different matter had Eliot argued that the CEP is a tautology, a logical truth, or $a$ priori true (Peters 1976), but he clearly believes that this is not the case (see Eliot 2011: 5).

The second way is to demand that ecologists develop systematic and general theoretical resources capable of explaining away the known or existing exceptions to the CEP as apparent exceptions. Weber (1999) claims that ecologists do have such resources, but he provides no argument to support this claim. Instead, Weber presents a few case studies showing how ecologists have explained away some of the exceptions to the CEP. Thus, Eliot (2011) is right in criticizing Weber for not providing an argument for there being systematic and general theoretical resources for ecologists to explain away the exceptions to the CEP. However, in this section we have provided systematic and general theoretical resources for ecologists to explain away the known exceptions to the CEP, i.e., the two coexistence mechanisms. In fact, even interfering conditions that may not belong to the domain of ecology can be explained away as apparent exceptions to the principle by means of these two coexistence mechanisms, showing that we have general and systematic theoretical resources to explain away the exceptions to the CEP as apparent exceptions.

\section{Causal Explanations in Biology}

To assess whether and how the CEP contributes to ecologists' explanations and causal understanding of phenomena, we adopt an interventionist framework of causal explanation, according to which explanatory regularities should remain invariant during their interventions (Woodward 2003b). Invariant regularities describe causal dependency relations, which can be used to manipulate things. In the interventionist account, causes are difference-makers in that they can be intervened upon to manipulate or control their effects. The causes and effects should be understood as being representable as variables. A change in the value of a cause thus makes a difference in the value of its effect.

An invariant regularity furnishes explanatory information by its ability to provide an answer to so-called what-if-things-had-been-different questions ("what if" questions), i.e., questions of what would happen to a value of the variable of a regularity if the value of one or more of its other variables were changed by an intervention or manipulation. Here, the stability of a regularity under interventions in its variables is what matters in explanations.

A necessary (although not sufficient) condition for a regularity to count as causally explanatory and invariant is that it expresses a change-relating relation. Change-relating regularities describe how changes in the value of their variable(s) are related to changes in the value of their other variable(s). For there to be an intervention and the possibility of a manipulation, at least some of the terms of a regularity are required to be representable as variables. There are various reasons why a regularity may count as non-change-relating, and thus causally non-explanatory: the terms of the regularity are too imprecise to be represented as variables with well-defined and manipulable values; the regularity denies changes in the properties of its terms; the regularity does not purport to represent a change-relating regularity in the first place, but, for instance, expresses a coexistence law (e.g., the Pauli exclusion principle); and so on.

Given this distinction between change-relating and non-change-relating regularities, invariance is a degree property of regularities with a threshold. Non-change-relating regularities are not invariant. Likewise, there are change-relating regularities, e.g., of correlations between factors that are joint effects of a common cause, that are not invariant. Accordingly, change-relating regularities that are invariant during interventions entail causally explanatory relevant information about dependency relations.

The interventionist account seems to accord well with the intuitions and practices of biologists with regard to experimentation and explanation (Park 1962). It resolves the problems of explanatory irrelevance and asymmetry. The account allows us to speak of absences and omissions as causes and consider preventions as effects unlike some other accounts of explanation, which is fortunate, since such negative facts are typically treated as causes and effects in the biological sciences. The account allows for explanations of phenomena that have low probabilities of occurrence in contrast to certain other accounts of 
explanation. The interventionist account thus provides the normatively right answers to many issues concerning explanations.

\section{Competition Theory, Causality, and Explanatory Power}

Eliot (2011: 5) argued against Weber (1999) that the CEP does not represent a cp law referring to a causal regularity, because the CEP is a functional or abstract regularity that "faces exceptions." We believe Eliot is right, but for the wrong reasons. We have already discussed why the CEP should not be interpreted as a cp law. In section 3, we argued that the CEP is a strict law that may be universally true. In this section, we discuss why the CEP should not be interpreted as a causal regularity. As we argue, the reason for the noncausal explanatory status of the CEP does not have to do with the CEP's functional or abstract status, in contrast to what Eliot (2011: 6-7) suggested, because abstract and functional regularities can be causally explanatory and even display high degrees of explanatory power.

What matters in assessing the causal explanatory status of the CEP is, moreover, not whether we can interpret the CEP as a law per se, but whether it shows invariance under interventions. It would be easy, but ill-advised, to argue for the non-causal status of the CEP as a non-change-relating and thus a non-causal regularity, because its terms are not representable as variables with well-defined values. Early Grinnellian and Eltonian definitions of niche as a space, position, or role of a species in nature's economy were too vague or general to be understood as variables with well-defined values. Similarly, there were difficulties in operationalizing the geometrical and abstract definition of niche by Hutchinson (1959), as an $n$-dimensional hypervolume, as a variable with well-defined, exact, and empirically testable values.

However, the modern definition of niche as a multidimensional utilization distribution that quantifies the species' or population's resource use along various axes is different and can be used to operationalize the Hutchinsonian niche concept (see Schoener 1989 and Griesemer 1992). It represents a niche as a variable with well-defined, exact, and empirically testable values, such as food particle size, ambient temperature, feeding mode/place/time, the size of the home range, and nutrient requirements.

In addition, the theory of limiting similarity with rules such as 1.3 and $\mathrm{d} / \mathrm{w} \approx 1$ provides examples of how to variabilize the related notion of "ecological dissimilarity" (i.e., there being important ecological differences between the niches of species), allowing coexistence between competing species with welldefined, exact, and empirically testable values. The rule $\mathrm{d} / \mathrm{w} \approx 1$ is about species packing in an environment or habitat. The niches of species are represented as utilization curves of their resources, whereby a utilization curve maps the frequency of using a resource. A species has a mean position in its utilization curve, and $d$ represents the distance between the means of two utilization curves of different species. A species' utilization curve also has a variance or standard deviation $(\mathrm{w})$ around the mean. Theoretical results and some data suggest that competitors could coexist if the niche overlap $d / w$ is approximately 1 . The 1.3 rule is a constant of limiting similarity between competing species. Hutchinson (1959) suggested that limiting similarity allowing coexistence can be measured by size, and some data supported the ratio of approximately 1.3 in a linear dimension of feeding structures as sufficient to permit coexistence.

As the above shows, both the niches of species and limiting similarity allowing coexistence can be represented as conceptually precise variables with well-defined and empirically testable values. The semantic accuracy of the terms included in the CEP also shows that the main argument by Peters (1976) and others against the CEP as not being an empirically respectful or testable statement or law, but rather a tautology, can be rejected. Moreover, the above indicates that the protective clause of the CEP, namely, that "species are able to coexist because there are important ecological differences between the niches of species or their use of resources," is not an empirically vacuous or ad hoc clause, since ecological differences can be given exact and empirically testable values as variables by applying the theory of limiting similarity. Consequently, when ecologists employ constitutive coexistence mechanisms as explanantia of exceptions to the CEP (see section 3), they do not rely on vague, tautological, or subjective ideas concerning what counts as an important ecological difference between species that allows for the coexistence of apparent competitors. Rather than being empirically vacuous, this part of the proviso clause of the CEP can be given an exact theoretical and conceptual content that can be empirically tested.

At the same time, we maintain that the CEP is neither an invariant nor a causal regularity. The reason is that the CEP is a coexistence law: a non-change-relating lawlike regularity. Change-relating regularities are 
uniformities of succession that describe regular ways in which events or states follow one another in time. They display the asynchronous characteristics of causal regularities. In other words, the hallmark of causation is succession in time. In general, change-relating regularities, such as Galileo's law and Newton's second law, are described by differential or difference equations that, given some initial conditions, can be used to calculate the values of a magnitude over time. In contrast, a law of coexistence expresses a relation concerning things or events that occur at the same time. It displays synchronicity in the dependence of the magnitudes involved. A coexistence law links two or more magnitudes by showing how their values are related to one another at the same time. In other words, they offer information on what regions of an $n$ dimensional state-space the system under study is (un)able to occupy at a certain time.

The traditional distinction between succession and coexistence laws is not perfectly coextensive with the distinction between change-relating and non-change-relating regularities by Woodward (section 4). First, the latter need not be lawlike regularities. Second, and more important, some authors have presented the Ideal Gas Law and the Law of The Pendulum as examples of coexistence laws, whereas, according to Woodward's criteria, these count as change-relating and even causal regularities. We do not want to debate whether these two and similar regularities are examples of coexistence laws as traditionally understood and defined, but rather we want to provide the term with a new and more precise definition: a coexistence law is a lawlike and non-change-relating regularity. ${ }^{3}$ According to this definition, the Ideal Gas Law and the Pendulum Law are not coexistence laws, but succession laws.

The CEP does not claim that certain changes in its terms lead to changes in its other terms later on. Rather than explaining the dynamics of a competitive system over time, the CEP restricts the states that the system could take by maintaining that the state of "n number of ecologically similar species with lower than n number of common limiting resources" cannot be maintained with another state in the system, namely, "stable coexistence." Rather than being a causal law, the CEP is a coexistence law or a non-change-relating and lawlike regularity stating that a certain result is not possible. Consequently, the CEP cannot contribute to the ecological explanation as a causal explanans. However, other parts of the competition theory fill this gap. $^{4}$

Among the classical causal generalizations in competition theory are the equations of the Lotka-Volterra competition model. The model consists of a system of coupled, non-linear differential equations. The system of equations presents the result of competition between species as a function of such factors as species' autecology and synecology (represented as different values of the coefficient of competition, $\alpha$ ) and the initial number of individuals, $\mathrm{N}$, of competing species. The model's equations include the following: the equation for the population growth of competitor species 1 is

$$
\mathrm{dN}_{1} / \mathrm{dt}=\mathrm{r}_{1} \mathrm{~N}_{1}\left(\left(\mathrm{~K}_{1}-\left(\mathrm{N}_{1}-\alpha_{12} \mathrm{~N}_{2}\right)\right) / \mathrm{K}_{1}\right)
$$

the equation for the population growth of competitor species 2 is

$$
\mathrm{dN}_{2} / \mathrm{dt}=\mathrm{r}_{2} \mathrm{~N}_{2}\left(\left(\mathrm{~K}_{2}-\left(\mathrm{N}_{2}-\alpha_{21} \mathrm{~N}_{1}\right)\right) / \mathrm{K}_{2}\right) \text {. }
$$

In these equations, $N_{1}$ is the number of individuals of species 1 at time $t ; N_{2}$ is the number of individuals of species 2 at time t. $K_{1}$ is the carrying capacity of species $1 ; K_{2}$ is the carrying capacity of species 2 . The $r_{1}$ is the intrinsic growth rate of species $1 ; r_{2}$ is the intrinsic growth rate of species 2 . The $\alpha$ 's are competition coefficients, where $\alpha_{12}$ is the negative effect of species 1 on species 2 , and $\alpha_{21}$ is the negative effect of species 2 on species 1 .

The equations in the model represent a modular system of invariant equations, and the model remains invariant during variations in the values of its independent variables $\left(\mathrm{N}_{1}\right.$ or $\left.\mathrm{N}_{2}\right)$, at least for certain parameter values. Thus, the Lotka-Volterra competition model is a causal explanatory model, because the equations (a) entail the right sort of counterfactual dependency relations (i.e., invariant relations) between independent and dependent variables of the model and because (b) they correctly describe, at least under certain interventions and background conditions, what would happen to the competitive system if we manipulated the values of the independent variables. Note also that (c) the terms in the Lotka-Volterra equations, such as $\mathrm{N}_{1}$ and $\mathrm{N}_{2}$, are variables with well-defined and changeable values. 
The Lotka-Volterra competition model is a system of abstract or functional generalizations that omit causally and mechanistically relevant details of competitive systems. The equations are thus phenomenological or non-mechanistic in not specifying the exact form of competition between species, such as interference or exploitative competition, nor do they consider resources or the specific form of resources, such as essential, substitutable, or hemi essential resources (Tilman 1980, 1986). Rather the model includes sufficient parameters, such as competition coefficients or carrying capacity. Sufficient parameters are abstract or general causal surrogates for various lower-level causes or mechanisms that seem to have the same or similar effects (Raerinne 2013).

The same holds for many explanations of exceptions to the CEP. Environmental heterogeneity is an example of an abstract causal surrogate. It includes various environmental factors - spatial and/or temporal that can prevent ecologically similar species from outcompeting one another. In other words, the various instances or realizations of environmental heterogeneity have robust results: they make apparent exceptions to the CEP possible. It is this robustness of results that justifies using sufficient parameters in models. Some mechanisms of environmental heterogeneity work by lowering population densities of competing species, which then lower the intensity of competition among species; others work by changing the competition rankings between the species and so on. But as a result of their robustness, those individual models that describe different causes or mechanisms of spatial or temporal environmental heterogeneity can be legitimately described with a simple abstract model with a sufficient parameter, such as environmental heterogeneity - a placeholder for a variety of different explanations of exceptions to the CEP.

Eliot (2011: 6-7) doubts that abstract mechanisms are capable of accounting for exceptions to the CEP, in contrast to what we have argued in section $3 .^{5} \mathrm{Next}$, we will show that both higher-level explanantia themselves and abstract or higher-level explanations of exceptions to a regularity can be explanatory and have high explanatory power without referring to lower-level mechanistic details as opposed to what is often suggested by authors who criticize the lack of mechanistic details in ecological models (see Schoener 1986; Denny \& Helmuth 2009).

There are two issues that must be distinguished: first, answering what it means for a higher-level causal explanation to count as explanatory at all and, second, addressing how good the explanations offered are. Higher-level explanantia (of exceptions) are explanatory if they provide the right sort of counterfactual dependency relations that remain invariant under their interventions (Woodward 2003a; Weber 2008). As we already argued above, the Lotka-Volterra model is an explanatory model according to this criterion. Yet, in the light of this model's lack of mechanistic detail, it can be asked how good the causal explanations are that the model offers to us. Does it and higher-level explanantia in general provide causal explanations with low explanatory values? This issue concerns the concept of explanatory power or goodness, not explanatoriness per se. With regard to this second issue, we maintain that lower-level explanations (of exceptions) are not always or simpliciter better as explanantia, because explanatory power is not a monolithic property of an explanation, and lower- and higher-level explanantia have different merits with regard to explanatory power.

Based on the interventionist framework adopted here, explanatory goodness can be evaluated by comparing the ranges of inferences to (potentially new) counterfactual situations, and thus the number and importance of answers to "what if" questions that rival explanations make possible. Sometimes we try to increase the number and importance of counterfactual inferences by sharpening the explanandum phenomenon, and thus calling for more mechanistic detail to be entailed in the explanans in order to address the refined explanandum.

In fact, this idea of making an explanation more precise is crucial for resource-based competition modelers seeking to provide mechanistic explications for the Lotka-Volterra competition model (Tilman 1980, 1986; Leibold 1995), as has also been discussed by Eliot (2011: 7-11). Here, causal surrogates such as the competition coefficient are reified by including specific details on the nature of the limiting resources in the model. For example, an explanans seeking to address the detailed explanandum of the distribution patterns of the competing insectivorous warbler species may consider a mechanism describing how climate influences the distribution of an important limiting resource of these species, such as arthropod prey (Gross \& Price 2000). This enables ecologists to make inferences about new counterfactual situations and to answer important questions such as "What would happen to the distribution of one or more bird species if we change the limiting factor(s) 'climate' and/or 'availability of insect prey'?” 
At the same time, supplying mechanistic detail alone does not amount to better explanations, since precision is not the sole factor that determines the goodness of an explanation. Goodness of explanation is constrained by at least two other factors (Ylikoski \& Kuorikoski 2010): cognitive salience and sensitivity. A phenomenon characterized too precisely can make an explanation too complex for us to understand. In other words, precision reduces cognitive salience.

In addition, a highly detailed explanation makes an explanatory relation more sensitive to changes in the values of explanans variables and/or of background conditions. This means that it continues to hold only under small numbers of these changes. For example, in the case of the warbler species mentioned above, the dependency between an explanans and a rather coarse-grained description of the bird species' distribution patterns is harder to disrupt than a dependency on an explanans with a fine-grained alternative explanandum that includes various details on the nature of limiting resources, such as essential or substitutable resources, feeding time, place, or mode, food particle size, and genetic make-up of different warblers, as well as information on abundance and distribution of the prey species' population(s), climatic conditions, and so on. The latter explanatory relation can be said to be more sensitive to changes. If an explanation - like the former, less detailed explanation - is less sensitive, then it is more powerful in that it enables inferences to more counterfactual situations in which the variables included in the explanans and/or background conditions take on non-actual values without breaking the explanatory relation. Thus, dependencies with less sensitivity carry more explanatory relevant information and are, as explanantia, more robust, since they give correct answers to larger sets of "what if" questions.

Given the dimensions of precision, cognitive salience, and sensitivity, the abstract Lotka-Volterra competition model and abstract explanations of exceptions to the CEP (see section 3) follow a special "lowprecision-but-low-sensitivity-and-high-cognitive-salience” strategy to claim explanatory power; choosing a less precise explanation and, thus, a rather coarse-grained or abstract and functional explanation with sufficient parameters enables these models to hold under a larger set of changes in the actual values of explanans variables (e.g., changes in the number of individuals or growth rate of competing species) and/or background variables (e.g., changes in the genetic make-up or phenotype of competing species) without breaking the explanatory relation. In addition, this particular type of explaining complex competitive scenarios is easier to grasp.

As this analysis shows, the replacement of abstract causal surrogates by more precise and lower-level causes or mechanisms is not the royal road to a better understanding of coexistence and competition in ecology. Once we adopt the multi-faceted concept of explanatory power, we see that sharpening the description of an ecological-scenario-to-be-explained and, accordingly, making the explanans more precise may damage our understanding of the phenomenon. This result is in line with recent analyses of reductionist explanatory strategies, e.g., in molecular biology and morphology, claiming that increasing the number of (lower-level) causes and mechanisms included in explanantia can be not only irrelevant, but even detrimental to the goodness of the explanations (Nathan 2012).

With regard to the CEP, our analysis shows that Eliot was right about the principle's non-causal status, but he errs about the reasons. Since the CEP is a non-change-relating lawlike regularity, it does not fit the interventionist criteria of causal explanation. Instead, the Lotka-Volterra competition model, a system of coupled invariant change-relating equations, does. In addition, despite the rather superficial or abstract character of the explanations offered by the Lotka-Volterra competition model and the higher-level explanations of exceptions to the CEP by coexistence mechanisms, these explanations have a high explanatory power in contrast to what was suggested by Eliot (2011).

\section{The Role of the CEP in Causal Explanation}

Eliot (2011: 11-14) suggested that there is a novel mode of explanation, called channeling explanation, functioning in competition theory, which is non-nomothetic in the sense that explanations are not dependent on deduction from laws:

An actual state of affairs is channel-explained when shown to remain possible in contrast with a range of other states of affairs which are shown [to be] impossible. States of affairs are shown [to be] impossible 
as a function of theory and/or information about an explanandum's circumstances .... The "channeling" aspect of such explanations is that, in a state-space, adding information about what is impossible channels states of affairs into remaining regions of viability (Eliot 2011: 10; original emphasis).

Eliot did not discuss the CEP in this context, but instead took up how related mechanistic recoursecompetition models (Tilman 1986) explain by channeling. Eliot claimed that adding information to the explanans provided by resource-competition models helps to narrow down regions of an $n$-dimensional state-space, which are inhabitable by individuals of a given species in a particular system. Thus, the channeling of explanations comes in degrees: adding information rules out regions of the state-space (i.e., alternative possibilities or states of affairs) in which viability is impossible and leaves increasingly fewer regions, which are (at least) "not impossible given available information" (Eliot 2011: 12; original emphasis).

We will try to give a positive reconstruction of Eliot's channeling explanations by first showing how these are compatible with the contrastive-causal account of explanation, and, second, showing how the CEP works in such explanations. It is surprising that Eliot did not discuss the CEP in this context, because the CEP provides a perfect constraining principle in channeling explanations.

Eliot's idea of channeling explanation is compatible with and more explicable in terms of a contrastive account of causal explanation in which both explanandum and explanans are contrastive (Weber 1999; Woodward 2003b; Ylikoski \& Kuorikoski 2010). Once we adopt this framework, the relationship between channeling explanation and causal explanation as well as the role of the CEP in causal explanation becomes manifest. According to this contrastive account, the claim " $x$ rather than $x^{*}$ explains $y$ rather than $y^{*}$ in the context $U^{\prime \prime}$ can be represented as " $x\left[x^{*}\right]$ explains $y\left[y^{*}\right]$ in the context $U$," where $x$ and $y$ represent the explanans and explanandum, respectively, and $x^{*}$ and $y^{*}$ represent their contrast classes. This framework allows us to be as precise as possible about both explanantia and explananda by adding information about contrastive non-actual "states of affairs" to an explanation. Eliot's idea is that channeling explanations provides us with information as to what are possible and impossible dynamics, states, or behaviors of a system. In other words, these explanations are able to explain, because they provide crucial information about the contrast classes of the explanans and/or explanandum, which helps to make explanations more precise. Thus, the idea of channeling explanations should perhaps not be described as novel, because the basic tenet can be expressed by means of the contrastive account of causal explanation: providing restrictions on the contrast classes helps make explanations better, because it allows us to be more specific about what is being explained and by what the former is being explained.

Within the contrastive framework of causal explanation, the CEP does not work directly as a causal explanans, because it is a coexistence law. At the same time, the CEP works in causal explanatory practices as a restrictive nomic principle. Following Weber (1999: 82-87), the "working area" of the CEP should be located in the contrast classes of explananda rather than explanantia. Here, the CEP works, first, restrictively by stating that some states of a competitive system are not possible: it rules out the possibility that stable coexistence of competitor species can be maintained when the common resources are limiting and fewer in number than the competitor species. Secondly, this restrictive information about the explanandum guides ecologists heuristically in searching for intervening and constitutive coexistence mechanisms that account for the failures of the CEP. Thus, the CEP helps to construct (part of) the contrast class of the explanandum and also helps to sharpen our understanding of what should be explained and how this could be explained.

Rather than working as a causal explanans, the CEP thus works as a nomic principle, guiding and sharpening causal explanation about competitive systems. The restrictive status of the CEP is secured by the fact that the principle has the nomic necessity of a strict law that is not riddled with genuine exceptions and may even be universally true. This nomic necessity justifies the restrictive use of the CEP and guarantees its non-causal, nomothetic explanatory potential.

In addition, the above shows that laws play an important role in (causal) explanatory practices in ecology, because the CEP is able to demarcate between states of affairs that are impossible or possible for competitive systems. This is in contrast to Eliot (2011), who denies the CEP the status of a law and, in general, seems to doubt that biological laws function in channeling explanations. According to Eliot (2011: 
1), the theoretical success of ecology can perhaps be assessed by evaluating whether its "theory might be epistemically robust despite having a different, lawless structure” (emphasis added).

\section{Conclusions}

In the literature, various ideas about the CEP's lawlike and explanatory status have been suggested. In contrast to Eliot (2011), we argued that the CEP is not riddled with exceptions, since most, and perhaps even all, of its exceptions are apparent, not genuine. Moreover, we claimed that the problem of a proviso clause of the CEP is not a semantic problem, but an epistemic one, which suggests that the CEP should not be interpreted as a ceteris paribus law, but as an empirically respectful and testable strict law, one that may even be universally true, pace Weber (1999) and Eliot (2011). This means that the CEP's proviso clause is not empirically vacuous, open-ended, or semantically inaccurate, but rather, it is a clause that can be strictly complemented. There are conditions under which the CEP's proviso clause holds, the CEP can be tested, and in which there is at least the possibility that genuine exceptions may be found to the CEP.

In addition, we argued that discussion about the CEP's causal explanatory status has focused on the wrong target and irrelevant features, such as the CEP's lawlike status. Since the CEP is a non-changerelating regularity or a coexistence law, it cannot causally explain. However, other invariant and changerelating regularities in competition theory serve this explanatory role. Finally, the CEP plays a role in the context of causal explanation. Rather than being a causal explanans, it acts as a non-causal restrictive principle with which causal explanations are contrasted and thus made more precise. The principle's own explanatory status can be stated in traditional terms: it offers non-causal, covering law explanations.

Moreover, the above suggests a more general conclusion: accounts of causal explanation in biology have neglected some of the important roles that non-causal laws play in causal explanations by facilitating, sharpening, or constraining these explanations. Accordingly, the theoretical success of biology perhaps cannot be assessed without some laws working in the background.

\section{Literature cited}

Amarasekare, P. 2007. Trade-offs, temporal variation, and species coexistence in communities with intraguild predation. Ecology 88: 2720-2728.

Carrier, M. 1998. In defense of psychological laws. International Studies in the Philosophy of Science 12: 217-232.

Chesson, P. 2000. Mechanisms of maintenance of species diversity. Annual Review of Ecology and Systematics 31: 343-366.

Chesson, P. and Huntly, N. 1997. The roles of harsh and fluctuating conditions in the dynamics of ecological conditions. American Naturalist 150: 519-553.

Davidson, D. 1970: Mental events. In Experience \& Theory. Ed. by L. Foster and J.W. Swanson. Massachusetts. University of Massachusetts Press, 79-101.

Denny, M. and Helmuth, B. 2009. Confronting the physiological bottleneck: A challenge from ecomechanics. Integrative and Comparative Biology 49: 197-201.

Durán, L.R. and Castilla, J.C. 1989. Variation and persistence of the middle rocky intertidal community of central Chile, with and without human harvesting. Marine Biology 103: 555-562.

Eliot, C.H. 2011. Competition theory and channeling explanation. Philosophy \& Theory in Biology 3: 1-16.

Fodor, J. 1991. You can fool some of the people all of the time, everything else being equal; Hedged laws and psychological explanations. Mind 100: 19-34.

Gilbert, O., Reynoldson, T.B. and Hobart, J. 1952. Gause’s hypothesis: An examination. Journal of Animal Ecology 21: 310-312.

Griesemer, J.R. 1992. Niche: Historical perspectives. In Keywords in Evolutionary Biology. Ed. by. E.F. Keller, and E.A. Lloyd: Cambridge: Harvard University Press, 231-240.

Gross, S.J. and Price, T.D. 2000. Determinants of the northern and southern range limits of a warbler. Journal of Biogeography 27: 869-878. 
Heatwole, H. and Davis, D.M. 1965. Ecology of three sympatric species of parasitic insects of the genus Megarhyssa (Hymenoptera: Ichneumonidae). Ecology 46: 140-150.

Hutchinson, G.E. 1959. Homage to Santa Rosalia, or why are there so many kinds of animals? American Naturalist 93:145-159.

Hutchinson, G.E. 1961. The paradox of the plankton. American Naturalist 95:137-145.

Kuorikoski, J. 2007. Explaining with equilibria. In Rethinking Explanation. Ed. by J. Persson and P. Ylikoski. Dordrecht: Springer, 149-162.

Lack, D. 1945. The ecology of closely related species with special reference to cormorant (Phalacrocorax carbo) and shag (P. aristotelis). Journal of Animal Ecology 14: 12-16.

Lack, D. 1946. Competition for food by birds of prey. Journal of Animal Ecology 15: 123-129.

Lange, M. 2005. Ecological laws: What would they be and why would they matter? Oikos 110: 394-403.

Leibold, M.A. 1995. The niche concept revisited: Mechanistic models and community context. Ecology 76: 1371-1382.

Leslie, P.H., Park, T. and Mertz, D.B. 1968. The effect of varying the initial numbers on the outcome of competition between two Tribolium species. Journal of Animal Ecology 37: 9-23.

Nathan, M.J. 2012. The varieties of molecular explanation. Philosophy of Science 79: 233-254.

Paine, R.T. 1966. Food web complexity and species diversity. American Naturalist 100: 65-75.

Park, T. 1948. Experimental studies of interspecies competition. I. Competition between populations of the flour beetles, Tribolium confusum Duval and Tribolium castaneum Herbst. Ecological Monographs 18: 266-307.

Park, T. 1962. Beetles, competition, and populations. Science 138: 1369-1375.

Pietroski, P. and Rey, G. 1995. When other things aren't equal: Saving ceteris paribus laws from vacuity. British Journal for Philosophy of Science 46: 81-110.

Peters, R.H. 1976. Tautology in evolution and ecology. American Naturalist 110: 1-12.

Raerinne, J. 2013. Robustness and sensitivity of biological models. Philosophical Studies 166: 285-303.

Schoener, T.W. 1986. Mechanistic approaches to community ecology: A new reductionism. American Zoologist 26: 81-106.

Schoener, T.W. 1989. The ecological niche. In Ecological Concepts. Ed. by J.M. Cherrett. London: British Ecological Society/Blackwell, 79-113.

Slobodkin, L.B. 1964. Experimental populations of Hydrida. Journal of Animal Ecology 33 (Suppl.): 131148.

Sober, E. 1983. Equilibrium explanation. Philosophical Studies 43: 201-210.

Tang, J. and Zhou, S. 2011. The importance of niche differentiation for coexistence on large scales. Journal of Theoretical Biology 273: 32-36.

Tilman, D. 1980. A graphical-mechanistic approach to competition and predation. American Naturalist 116: 362-393.

Tilman, D. 1986. A consumer-resource approach to community structure. American Zoologist 26: 5-22.

Utida, S. 1953. Interspecific competition between two species of bean weevil. Ecology 34: 301-307.

Weber, M. 1999. The aim and structure of ecological theory. Philosophy of Science 66: 71-93.

Weber, M. 2008. Causes without mechanisms: Experimental regularities, physical laws, and neuroscientific explanation. Philosophy of Science 75: 995-1007.

Woodward. J. 2003a. Experimentation, causal inference, and instrumental realism. In The Philosophy of Scientific Experimentation. Ed. by H. Radder. Pittsburgh: University of Pittsburgh Press, 87-118.

Woodward, J. 2003b. Making Things Happen. Oxford: Oxford University Press.

Ylikoski, P. and Kuorikoski, J. 2010. Dissecting explanatory power. Philosophical Studies 148: 201-219.

\section{Notes}

1. Our way of characterizing cp laws is not meant to subscribe to any specific account of cp laws. Rather, we framed the idea of cp laws as simply as possible in order to make certain points about explanations of exceptions, various exceptions to the laws, etc. There are other ways to include cp clauses in statements of laws besides placing them in the antecedents. Nevertheless, the other ways are often logically 
equisatisfiable. Additionally, the problems of the cp law account are independent of the various formal ways used to express or include cp clauses in law statements.

2. Lange (2005) argued that ecologists need not be concerned about all the interfering conditions that are physically possible and that might violate ecological laws. This suggests that many of the abovementioned interfering conditions are outside the domain of counterfactual scenarios about which ecologists should be concerned. However, let us suppose that this is not the case. Moreover, there are convincing theoretical arguments to show that these interfering conditions do not decrease competitive interactions per se, but affect only the rate at which competitive exclusion is reached (Chesson \& Huntly 1997; Chesson 2000). Thus, interfering conditions are not sufficient as genuine exceptions to the CEP. This suggests that the principle is valid and perhaps even universally so, because there is no coexistence without important niche differences between competing species. Let us, however, suppose that this, too, is not the case. The reader may have observed that we have made our own argument for the strict lawlike nature of the CEP much more difficult than was necessary. The above two instances of "let us suppose these are not the cases" would already have given us arguments to show that the CEP is not riddled with unsystematic and unexplainable exceptions, contrary to what Eliot suggests, because the I's in question are outside the domain of ecology and ecologists need not be concerned about the I's to begin with (Lange 2005) and/or the I's produce only apparent exceptions to the CEP (Chesson \& Huntly 1997, Chesson 2000). Our point, however, is that if we can provide an argument for the strict lawlike nature of the CEP under the conditions in which various kinds of I's, inside or outside the domain of ecology, produce only systematic and explainable apparent exceptions to the CEP, then we have a much stronger argument against the ideas that the CEP is riddled with genuine unsystematic exceptions and that ecologists do not have the theoretical resources needed to explain away the exceptions to the CEP, which seem to be the main reasons that Eliot (2011) denies the lawlike status of the CEP.

3. Note that not all non-change-relating regularities count as lawlike or coexistence laws according to this definition: if the regularity is non-change-relating because its terms are imprecise, untestable, etc., then the regularity should not be counted as a law, because laws, among other things, should have precise truth-values, be testable, and so on. We already argued above that this is not the case with respect to the CEP, pace Peters (1976).

4. An anonymous referee asked whether the following scenario counts as an intervention in the CEP and thus provides a counterexample to our interpretation of the CEP as a non-causal coexistence law. A second species (with a niche sufficiently similar to that of the first species) is introduced into a (sufficiently uniform, sufficiently resource-limited) environment, which then, according to the CEP, and given sufficient time, leads to the situation that only one species, the better competitor, will remain. In other words, we add a second ecologically similar species to a system that originally contained only one species and one limiting resource; thus, the system is changed from a non-competitive to a competitive system. In this case, the CEP does not describe the first system at all. Thus, this cannot be an intervention in the CEP. In addition, after the system has been changed to a competitive one by the intervention, the CEP holds and is not changed. Thus, the counterexample fails. Moreover, one may ask whether the CEP provides equilibrium explanations to explain the dynamics of competitive systems. Thus, the CEP would be a succession law rather than a coexistence law. It is not the CEP, but rather the competition models that explain or describe how competition systems evolve over time, what the systems' population dynamics are, toward which stable attractors or stable equilibrium points the systems evolve, and so on. Many, but not all, of the competition models provide equilibrium explanations. In addition, to reply to a somewhat contrasting concern of another referee, this equilibrium aspect of competition models provides no difficulties for their causal explanatory status, as was suggested by Sober (1983). Woodward's account has no difficulties in accommodating equilibrium explanations as causal (see Kuorikoski 2007).

5. Eliot only hints at why he doubts the explanatory status of abstract mechanisms in explaining away the exceptions to the CEP. One reason he mentions (Eliot 2011: 13) is that adding incorporating distributing conditions to abstract competition models, such as Lotka-Volterra models, would amount to models that are so complex as to become intractable. This is not a problem for abstract models. These models can incorporate the complexity by means of sufficient parameters that guard against intractability. In fact, 
Eliot's favorite mechanistic competition models, such as Tilman's, are more vulnerable to the concern that incorporating distributing conditions into the model begets the model's intractability, because adding mechanistic details to a model itself often makes the model more complex and intractable.

\section{ACKNOWLEDGMENTS}

The work was supported financially by the Academy of Finland as part of the project Causal and Mechanistic Explanations in the Environmental Sciences (project no. 1258020) (Jani Raerinne), as well as by the Foundation Mercator and the Ruhr University Research School (Jan Baedke). We are grateful to two anonymous referees who provided critical, sharp-eyed, and constructive comments on previous drafts of the article. The editor, Jonathan Kaplan, also kindly provided helpful comments on previous versions of this article.

Copyright (C) 2015 Author(s).

This is an open-access article distributed under the terms of the Creative Commons Attribution-NonCommercial-NoDerivs license, which permits anyone to download, copy, distribute, or display the full text without asking for permission, provided that the creator(s) are given full credit, no derivative works are created, and the work is not used for commercial purposes.

ISSN 1949-0739 